\title{
Investigating shakeoff process in precise correlation measurements in nuclear $\beta$ decay
}

\author{
E. Liénard ${ }^{1[0000-0003-3748-4021]}$, P. Delahaye ${ }^{2[0000-0002-8851-7826]}, \mathrm{X}$. \\ Fléchard $^{1[0000-0003-3754-9083]}$, B. Pons ${ }^{3[0000-0002-0001-7107]}$ and G. Quéméner ${ }^{1[0000-0001-6703-6655]}$ \\ ${ }^{1}$ Normandie Univ, ENSICAEN, UNICAEN, CNRS/IN2P3, LPC Caen, 14000 Caen, France \\ ${ }^{2}$ GANIL, CEA/DSM-CNRS/IN2P3, Bd Henri Becquerel, 14000 Caen, France \\ ${ }^{3}$ Université de Bordeaux - CNRS - CEA, CELIA, UMR5107, F-33405 Talence, France \\ lienardelpccaen.in2p3.fr
}

\begin{abstract}
Precise measurements of correlations in nuclear $\beta$ decays are currently employed to probe the Standard Model. When the $\beta$ particle and recoiling daughter ion are detected in coincidence, these measurements further allow the observation of the ion charge-state distribution which results from the atomic shakeoff process induced by the nuclear decay. This is of great interest for fundamental atomic physics. This paper presents the results obtained at GANIL by means of LPCTrap experiments with ${ }^{6} \mathrm{He}^{1+},{ }^{35} \mathrm{Ar}^{1+}$ and ${ }^{19} \mathrm{Ne}^{1+}$ ions. Comparison with theoretical values is also presented and suggests further investigations.
\end{abstract}

Keywords: Ion trapping, Correlations in nuclear $\beta$ decay, Shakeoff process.

\section{Introduction}

\subsection{Correlations in nuclear $\beta$ decay}

Precision measurements in nuclear $\beta$ decay constitute sensitive probes of the Standard Model of elementary particles (SM), complementarily to high energy physics experiments [1]. Correlations between particle momenta and/or spins enable to test the violation of fundamental symmetries or the V-A structure of the SM, according to the behavior of vectors involved in the correlation term under symmetry operations ( $\mathrm{P}$ and $\mathrm{T})$. In particular, the $\beta-v$ angular correlation term, $a_{\beta v} \frac{\vec{p}_{p} \cdot \vec{p}_{v}}{E_{e} E_{v}}$, is invariant under $\mathrm{P}$ and $\mathrm{T}$ operations and enables either to search for exotic scalar or tensor currents beyond the V-A theory in pure Fermi(F) or Gamow-Teller (GT) transitions, or to determine precisely the mixing ratio between the GT and F parts in mirror transitions.

Since neutrinos are difficult to detect, sensitive observables for $\beta-v$ angular correlation measurements are kinematic parameters affected by the recoiling daughter nucleus motion. Two methods are commonly used: (i) a direct method in which either the recoil energy distribution [2] or the recoil ion time of flight (ToF) distribution thanks to its detection in coincidence with the $\beta$ particle [3], is measured or (ii) an indirect method in which the Doppler shift of a delayed particle ( $\gamma$ or $p$ ) emitted during the recoil motion [4-6] is measured. The direct method requires the use of very clean radioactive sources, 
ideally placed in vacuum, due to the very low recoil kinetic energy ( $\sim 1-2 \mathrm{keV}$ at most). The development of intense radioactive beams in new generation facilities coupled to efficient beam handling techniques has allowed the use of atom or ion traps to define excellent radioactive sources in very clean environment [3,7-9]. However, the high precision level targeted $(\sim 0.1 \%)$ requires a deep knowledge of the experimental setup to properly manage any effect potentially affecting the shape of the recoil energy or ToF distribution. The analysis of data is performed with the help of realistic simulations considering a set of systematic effects as complete as possible [10,11]. For instance, the effect due to the shakeoff process has to be considered since it modifies the charge state of the recoil ion. This process is introduced in the next section.

\subsection{The shakeoff process}

Electron shakeoff (SO) is a fundamental atomic process in which a bound electron is excited into the continuum because of a sudden change of the central potential. Nuclear $\beta$ decay, which modifies the nuclear charge, may induce such an ionization process. If one neglects the SO process, the $\beta$ decay of singly charged ions basically leads to:

$$
\begin{array}{ll}
\beta^{-}: & { }_{Z}^{A} X^{1+} \rightarrow \underset{Z+1}{A} Y^{2+}+\beta^{-}+\bar{v}_{e} \\
\beta^{+}: & { }_{Z}^{A} X^{1+} \rightarrow{ }_{Z-1}^{A} W^{0}+\beta^{+}+v_{e}
\end{array}
$$

As low energy neutrals are very difficult to detect, it is worth noting that in the second case, the recoil atom cannot be properly detected, while SO, yielding singly- and multicharged ions $W^{\mathrm{q}+}$, greatly facilitates the measurement. It is even worse with atom traps in which the radioactive source is neutral: detection of recoils in $\beta^{+}$decay then requires a double SO process [12]. The probability of multiple SO processes depends obviously on the number of electrons available but also on the probability of other atomic processes such as electron shakeup (excitation) or Auger emission. This finally leads to charge state distributions that should emphasize the dominant atomic processes.

The $\beta$ decay is a very fast process inducing a nuclear potential change with a typical time of $10^{-18} \mathrm{~s}$, which justifies the use of the sudden approximation (SA) in the theoretical models describing SO. However this hypothesis had not been yet experimentally verified before the first experiment performed at GANIL with the LPCTrap setup described in the next section.

\section{$2 \quad$ LPCTrap@GANIL}

The LPCTrap setup is installed at the low-energy beam line LIRAT of the SPIRAL1GANIL facility. Technical details on the setup are given in [13]. The central element of the device is a transparent Paul trap designed to confine singly charged radioactive ions, almost at rest, in a small volume. The trap consists of 6 concentric rings with an open geometry, allowing easy injection and extraction as well as an efficient detection in coincidence of the $\beta$ particles and recoil ions.

The low energy radioactive beam is provided by the ECR source of the SPIRAL facility at $10 \mathrm{keV}$ kinetic energy (typical energy dispersion: $\sim 20 \mathrm{eV}$ ). In the LIRAT 
beam line, the installation is equipped with a Radio Frequency Cooler-Buncher (RFQCB) for the preparation of the beam by reducing the emittance and the production of ion bunches. In the transparent Paul trap, the ions, also cooled down by elastic collisions with an inert gas injected at very low pressure $\left(10^{-6}-10^{-5} \mathrm{mbar}\right)$, have energies of about $0.1 \mathrm{eV}$ at equilibrium and the diameter of the ion cloud located at the centre of the trap is of the order of $2.5 \mathrm{~mm}$.

In the detection setup, a telescope and a micro-channel plate position sensitive detector (MCPPSD) detect the $\beta$ particles and the recoil ions respectively. The $\beta$ telescope, located $10 \mathrm{~cm}$ away from the center of the trap, is made of a $60 \times 60 \mathrm{~mm}^{2} 300 \mu \mathrm{m}$ thick double-sided silicon strip detector for position readout, followed by a $7 \mathrm{~cm}$ thick plastic scintillator coupled to a photomultiplier to measure the $\beta$ particle kinetic energy and to deliver the start signal for the recoil ion ToF measurement. The stop signal is provided by the MCPPSD, installed at the end of the ion spectrometer. This spectrometer is located in front of the telescope at the opposite side from the trap center. The ions emitted towards the spectrometer are accelerated by a $-2 \mathrm{kV}$ potential after they cross a collimator located in front of a $50 \mathrm{~cm}$ long free flight tube. An electrostatic lens allows the collection of all the ions on the MCPPSD. This spectrometer makes LPCTrap a unique and unprecedented setup for the measurement of charge-state distributions of ions associated to the $\beta$ decay of singly charged radioactive ions. The performances of the whole setup for experiments performed with ${ }^{6} \mathrm{He},{ }^{35} \mathrm{Ar}$ and ${ }^{19} \mathrm{Ne}$ are given in [14].

\section{The ${ }^{6} \mathrm{He}^{1+}$ case}

According to equation (1), one single electron remains bound to the ${ }^{6} \mathrm{Li}^{2+}$ recoil ion after the $\beta^{-}$decay of ${ }^{6} \mathrm{He}^{1+}$. Therefore the final ion charge-state distribution only depends on pure SO, and the basic assumptions underlying the usual descriptions of SO can be checked precisely by means of direct comparison with experiment. Full quantum calculations, employing hydrogen-like wavefunctions, were thus performed in the framework of the SA with the aim to probe the reliability of this latter fundamental approximation. The calculations led to the SO probability (see [15] for details): $\mathrm{P}_{\mathrm{SO}}=\left(2.33810+0.00412 E_{\text {rec }}(\mathrm{keV})\right) \times 10^{-2} \quad$ where $E_{\text {rec }}$ is the recoil kinetic energy.

Figure 1 shows the recoil ion ToF distribution measured at GANIL using LPCTrap. A total of $1.2 \times 10^{6}$ final coincidences has been collected and analyzed with realistic simulations including all the identified systematic effects [15]. The induced corrections and systematic uncertainties are summarized in Table 1. In the simulations, the SM value was used for $a_{\beta v}$, considering a variation of the parameter in a range compatible with the current experimental precision [16]. The difference in MCP efficiency due to different thresholds for the two charge states was also taken into account. The agreement between the simulated and experimental distributions is excellent and it has enabled to deduce the following experimental SO probability:

$\mathrm{P}_{\text {SO }}=0.02339(35)_{\text {stat }}(07)_{\text {syst }}$.

The mean recoil energy corresponding to the events selected in the experimental data analysis has been substituted in the theoretical expression to compute the mean SO probability. A correction to the SA has been further estimated to account for the finite duration of the potential change, including also the direct collision mechanism between 
the bound electron and $\beta$ particle, yielding $\delta \mathrm{P}_{\mathrm{SO}}=-20 \times 10^{-5}$. Finally, the mean theoretical SO probability is $\left\langle\mathrm{P}_{\mathrm{SO}}\right\rangle=0.02322$, in perfect agreement with the experimental value.

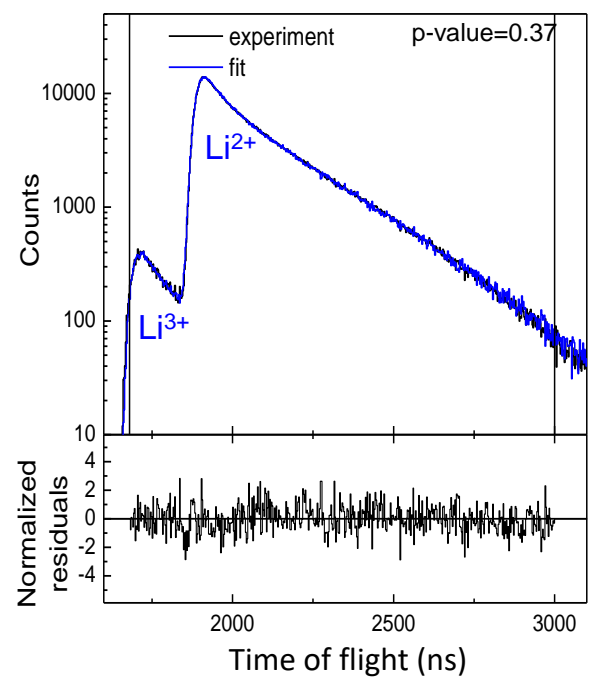

Fig. 1. Experimental ${ }^{6} \mathrm{Li}$ ions ToF distribution following ${ }^{6} \mathrm{He}^{1+}$ decay, with the fit superimposed (adapted from [15]).

Table 1. Systematic effects considered in the analysis. (See text for details).

\begin{tabular}{cccc}
\hline Source & Corr. $\left(10^{-5}\right)$ & Error $\left(10^{-5}\right)$ & Method \\
\hline$a_{\beta \nu}$ & & 4.0 & {$[16]$} \\
$\beta$ scattering & 39 & 4.0 & GEANT4 \\
Background & & 3.5 & present data \\
E calibration & & 1.7 & present data \\
MCP efficiency & -9 & 1.2 & present data \\
Total & 30 & 7.0 & \\
\hline
\end{tabular}

\section{The ${ }^{35} \mathrm{Ar}^{1+}$ case}

According to equation (2), the recoiling ${ }^{35} \mathrm{Cl}$ species are neutral after the $\beta^{+}$decay of ${ }^{35} \mathrm{Ar}^{1+}$ ions but the SO process allows easier and direct detection of the recoil ions. Multielectron systems require a more sophisticated model to understand the chargestate distribution measured in the experiment. The calculation is performed in the framework of the SA, using an independent particle model (IPM) with Hartree-Fock wavefunctions to compute the ionization SO probabilities [17]. Subsequent Auger decays are also explicitly included in the calculation, and we discriminate between SO and Auger contributions to the production of charged daughter ions.

Figure 2 (Left) shows the recoil ion ToF distribution measured at GANIL using LPCTrap [17]. A total of $1.5 \times 10^{6}$ final coincidences has been collected. The experi- 
mental relative SO probability, indicated on the spectrum, was obtained by peak integration including two corrections: the overlap of peak tails deduced from basic simulations (assuming SM $a_{\beta v}$ ) and the charge dependence of the recoiling ion detection efficiency. The comparison with the model is again excellent when including Auger emission (see Fig.2 Right), and this confirms the paramount importance of Auger contribution to the production of high charge states. Moreover, the number of ${ }^{35} \mathrm{Cl}$ atoms produced during the experiment has been estimated using the number of detected $\beta$ particles reduced by the number of coincidences corrected by the overall absolute detection efficiency for ions. This estimation leads to $72(10) \%$ of neutral ${ }^{35} \mathrm{Cl}$, which is also in good agreement with the $73.9 \%$ ratio obtained from the calculation.
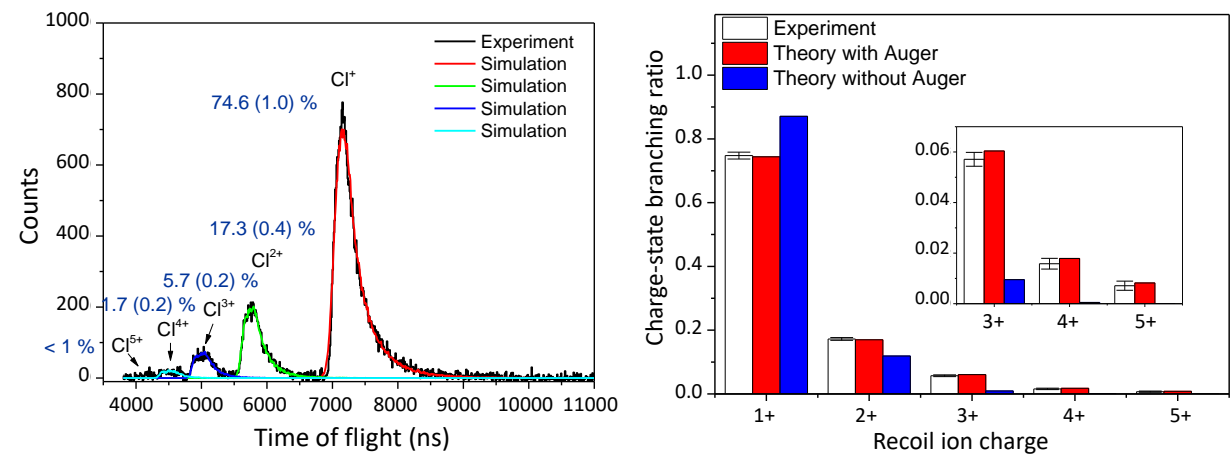

Fig. 2. Left: Experimental ${ }^{35} \mathrm{Cl}$ ions $\mathrm{ToF}$ distribution following ${ }^{35} \mathrm{Ar}^{1+}$ decay, with the simulated distributions superimposed (adapted from [17]). Right: Comparison between experimental and theoretical values with and without Auger emission (adapted from [18]). (See text for details)

\section{$5 \quad$ The ${ }^{19} \mathrm{Ne}^{1+}$ case}

The last case studied with LPCTrap concerns the charge-state distribution of the recoiling ${ }^{19} \mathrm{~F}$ ions after the $\beta^{+}$decay of ${ }^{19} \mathrm{Ne}^{1+}$ ions [18]. A total number of $1.3 \times 10^{5}$ final coincidences has been collected in the spectrum shown in Fig.3 (Left). The data were analyzed using the same method as for ${ }^{35} \mathrm{Cl}$ and the theoretical relative SO probabilities were obtained using the same approach as before. Here, the agreement seems better when Auger emission is neglected (see Fig.3 Right), which is not satisfactory and clearly coincidental. The production of neutrals, experimentally estimated to be $69.5(4.2) \%$, is also badly reproduced by the model which provides a value of $76.5 \%$. We traced back the root of the experimental/theoretical discrepancies to the inaccuracy of the multielectron SO probabilities computed by means of the IPM whose reliability worsens for systems with low nuclear charge [18]. 

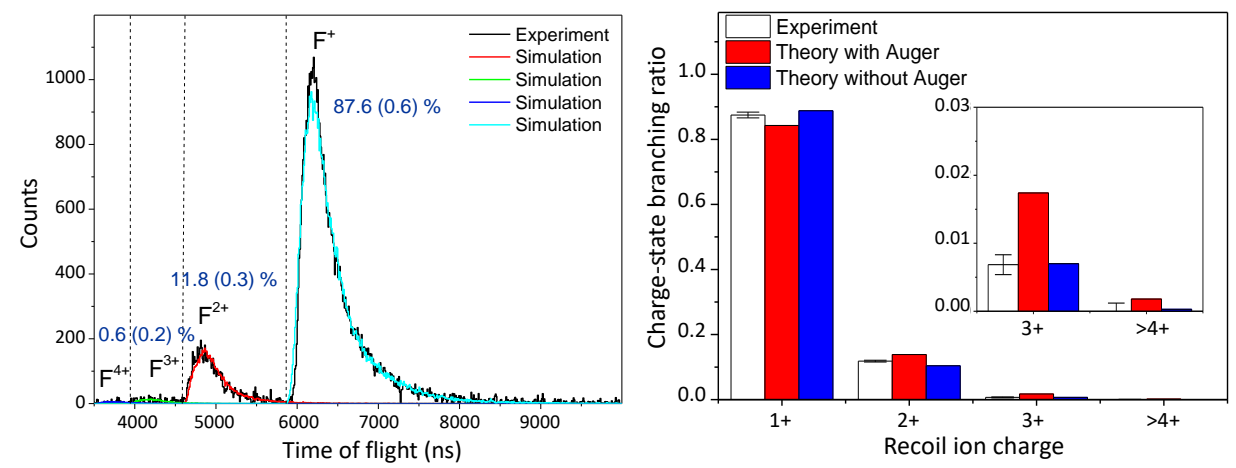

Fig. 3. Left: Experimental ${ }^{19} \mathrm{~F}$ ions ToF distribution following ${ }^{19} \mathrm{Ne}^{1+}$ decay, with the simulated distributions superimposed. Right: Comparison between experimental and theoretical values with and without Auger emission. (See text for details) The figures are adapted from [18].

\section{Conclusion}

The SO process has been investigated in three nuclear $\beta$ decays, starting from an ideal one-electron system which has enabled to confirm for the first time the reliability of the sudden approximation. In systems involving many electrons, primary SO ionization is followed by secondary processes which modify the recoil charge-state distribution. In ${ }^{35} \mathrm{Ar}^{1+}$ decay, Auger emission is dominant and monitors the production of the highest charge states, which is not observed in ${ }^{19} \mathrm{Ne}^{1+}$ decay. In this last case, Auger emission does not play such an important role so that the theoretical/experimental discrepancies which have been observed reveal the importance of electron correlations in the primary SO mechanism, beyond the independent electron assumption. Introducing such correlations in the calculation of SO probabilities constitutes a theoretical challenge and their importance as function of the nuclear charge $\mathrm{Z}$ could be directly gauged in future LPCTrap experiments performed at GANIL using beams $\left({ }^{21} \mathrm{Na},{ }^{23} \mathrm{Mg},{ }^{33} \mathrm{Cl},{ }^{37} \mathrm{~K}\right)$ provided by the new SPIRAL1 sources [19].

\section{References}

1. González-Alonso, M., Naviliat-Cuncic, O., Severijns, N., Prog. Part. Nucl. Phys. 109, 165223 (2019).

2. Johnson, C.H., Pleasonton, F., Carlson, T.A., Phys. Rev. 132, 1149-1165 (1963).

3. Gorelov, A. et al., Phys. Rev. Lett. 94, 142501 (2005).

4. Egorov, V. et al., Nucl. Phys. A 621, 745-753 (1997).

5. Adelberger, E.G. et al., Phys. Rev. Lett. 83, 1299-1302 (1999).

6. Severijns, N., Blank, B., J. Phys. G: Nucl. Part. Phys. 44, 074002 (2017).

7. Hong, R. et al., Phys. Rev. A 96, 053411 (2017).

8. Sternberg, M.G. et al., Phys. Rev. Lett. 115, 182501 (2015).

9. Fléchard, X. et al., Phys. Rev. Lett. 101, 212504 (2008). 
10. Fléchard, X. et al., J. Phys. G: Nucl. Part. Phys. 38, 055101 (2011).

11. Fabian, X. et al., Hyperfine Interact. 235, 87-95 (2015).

12. Scielzo, N.D., Freedman, S.J., Fujikawa, B.K., Vetter, P.A., Phys. Rev. A 68, 022717 (2003).

13. Ban, G. et al., Ann. Phys. (Berlin) 525, 576-587 (2013) and references therein.

14. Liénard, E. et al., Hyperfine Interact. 236, 1-7 (2015).

15. Couratin, C. et al., Phys. Rev. Lett. 108, 243201 (2012).

16. Glück, F., Nucl. Phys. A 628, 493-502 (1998).

17. Couratin, C. et al., Phys. Rev. A 88, 041403(R) (2013).

18. Fabian, X. et al., Phys. Rev. A 97, 023402 (2018).

19. Chauveau, P. et al., Nucl. Instrum. Methods Phys. Res. B 376, 35-38 (2016). 